\title{
Dynamical Processing of Stars and Planets Through Star Clusters
}

\author{
Aaron M. Geller*i \\ Center for Interdisciplinary Exploration and Research in Astrophysics (CIERA) and Dept. of \\ Physics and Astronomy, Northwestern University, 2145 Sheridan Rd, Evanston, IL 60208, USA \\ \& \\ Adler Planetarium, Dept. of Astronomy, 1300 S. Lake Shore Drive, Chicago, IL 60605, USA \\ E-mail: a-geller@northwestern.edu
}

\begin{abstract}
Most stars are born in clustered environments that are far denser than the Solar Neighborhood. Yet, star clusters can be hostile locations, where close stellar encounters can be frequent and can have violent consequences, including direct stellar collisions. Such encounters can dramatically alter stellar and planetary systems, and can produce exotic stars that define new pathways in stellar evolution. Understanding how, and to what extent, such "dynamically processing" occurs in star clusters may be critical for our understanding of the architectures of today's observed stellar and planetary systems, as well as the origins of X-ray sources, blue stragglers, sub-subgiants, and other stellar exotica. In this contribution, I discuss the impacts of living in a star cluster on its inhabitants, through a review of observational and theoretical efforts to study these complex systems. Throughout, I assert that binary stars are intimately linked to both the dynamical evolution of star clusters and the effects that stellar encounters within these environments have on their stellar and planetary populations. I conclude that in order to properly interpret our present and future observations of stars and planets, both in star clusters and the field, and particularly if we attempt to use these observations to inform star and planet formation theory, we must first account for the imprint left by stellar encounters within a clustered birth environment.
\end{abstract}

BASH 2015

18 - 20 October, 2015

The University of Texas at Austin, USA

\footnotetext{
* Speaker.

${ }^{\dagger}$ NSF Astronomy \& Astrophysics Postdoctoral Fellow
} 


\section{Introduction: Implications of Clustered Star Birth}

Any investigation into the origins of the present-day characteristics of binary (and higherorder) star systems and planetary systems must first consider the possible effects of being born in a star cluster. Within the Galactic field, stellar and planetary systems live essentially in isolation; only the very widest field systems (e.g., those with separations over $\sim 10^{3} \mathrm{AU}$ ) are in danger of encountering passing stars, or having their orbits changed dramatically by the Galactic tidal field [47]. However, most stars (at least those with masses $\geq 0.5 \mathrm{M}_{\odot}$ ) form in denser environments $[54,24,12]$.

More specifically, Spitzer surveys found that $90 \%$ of young stars live within clusters with $\geq 35$ members and stellar densities $\geq 1 \mathrm{M}_{\odot} \mathrm{pc}^{-3}$ [24]. Though only about a quarter of nearby young stars appear to be forming in dense clusters ( $\geq 200 \mathrm{stars}_{\mathrm{pc}}^{-2}$, projected) [12], observations also suggest that many clusters may form with substructure and also subvirial velocities. In other words, many very young cluster are "clumpy", peppered with regions of higher densities, and given the present observed velocities, many young clusters are "cool" and collapsing. Following their dynamical evolution shows that initially cool and clumpy clusters can collapse (on of order a crossing time) to obtain a brief period of significantly higher densities [70, 31].

Within dense stellar environments, close encounters with other stars can be frequent enough to change the properties of a significant fraction of stellar and planetary systems from how they were born. Stellar dynamical encounters can have a wide range of outcomes, depending on the number and masses of stars involved, the characteristics and orientation of each system, the incoming velocity and impact parameter, etc., but in general, encounters can (i) completely disrupt a stellar/planetary system, (ii) exchange stars and planets into and out of the system, (iii) modify the orbital elements of the system, and/or (iv) lead to direct stellar (and planetary) collisions. All of these mechanisms "dynamically process" stellar and planetary systems, and can alter the aggregate properties of a population.

Finally, many young star clusters eventually dissolve to populate the Galactic field [1, 54]. Therefore, our interpretation of the characteristics of stellar and planetary systems both in star clusters and the field (as well as our understanding of star formation in general), relies on how a population of stars evolves through this more dynamically active early stage in a star cluster. Moreover, studying the properties of a population of stellar and planetary systems today may reveal imprints of past dynamical activity within their birth cluster.

\subsection{The Birthplace of Our Solar System}

Fred Adams wrote an excellent review in 2010 examining the birth environment of our Solar System [2]. Within that paper, he investigates numerous lines of reasoning that support the hypothesis that our Solar System formed in a modest star cluster, initially containing $10^{3}-10^{4}$ stars. Figure 7 in his paper, summarizes much of the analysis and the observational constraints on the Solar birth cluster. In short, the lower bound on the number of stars in our birth cluster is derived from the requirements that (i) a star (or stars) massive enough to undergo a supernova formed near enough to enrich the Solar nebula in order to explain the presence of short-lived radioactive isotopes as inferred from meteoritic data, and (ii) a stellar encounter at an impact parameter of $\lesssim 400 \mathrm{AU}$ was likely enough to occur in order to produce the orbit of Sedna. The upper bound is derived from 
the requirements that (iii) there were few enough massive stars nearby that the Solar nebula was not evaporated (or too severely truncated) by far-UV radiation, and (iv) a stellar encounter at an impact parameter of $\lesssim 250$ AU was unlikely to occur so that the planets' orbits are preserved. Adams combines these constraints to estimate that the Solar System formed in a cluster with initially 4300 \pm 2800 stars.

I mention this example in detail here because it is "close to home". Speaking more broadly, since the majority of stars are thought to form in clusters or groups, it is very reasonable to assume that the majority of planetary systems also form within star clusters or groups. The impact of this clustered birth environment is apparent in our own Solar System, and furthermore, the dynamics of this birth environment are (perhaps) imprinted on the orbit of Sedna (and possibly other bodies). Thus again, it is clear that the impacts of possible close stellar encounters within a clustered birth environment must be considered when interpreting observations of planetary (and stellar) systems, and especially when one uses these observations to inform star and planet formation theories,

Finally, I note that a full investigation of the dynamical impact of a star cluster containing $10^{3}-10^{4}$ stars on the evolution of multi-planet systems (including our own Solar System) requires direct and self-consistent $N$-body star cluster models, including a realistic binary- (and higherorder-multiple-) star population. This has yet to be attempted, as no appropriate code currently exists (though I am actively working on this problem!).

\subsection{Open and Globular Star Clusters}

Before proceeding, it is important to define a bit of nomenclature. Within our Galaxy we generally divide star clusters into two categories: open clusters and globular clusters. Below and in Table 1, I provide a brief definition of these two cluster types, as they relate to the subject matter of this review. I also point the interested reader to Chapter 1.1.4 in Binney \& Tremain's book on "Galactic Dynamics" [9], where they also provide a concise description of open and globular clusters.

Most of the stars in the Galactic disk are believed to have formed in clusters similar to the open clusters we observe today. However, many star clusters likely dissolve shortly after expelling their natal gas (thanks to OB star winds and supernovae), due to the rapid decrease in magnitude of the gravitational potential well. Indeed most star clusters are thought to dissolve at ages younger than $\sim 1$ Gyr [54]. Those that survive the embedded phase in our Galaxy are typically called open clusters.

Open clusters generally contain between roughly 100 to $10^{4}$ stars, and can have a wide range in ages. The mean age of the Galactic open clusters is only a few 100 Myr. However some open clusters are many Gyr old, and these systems in particular are highly dynamically evolved. In other words, these old open clusters have lived through many 10s of half-mass relaxation times (see Table 1), where the half-mass relaxation time is an estimate of the average time needed for energies to be redistributed between stars by long-distance encounters, and thereby to set up a near-Maxwellian velocity distribution throughout the cluster. There are a few 1000 observed open clusters that have been studied to various degrees [48], though some estimates of the total number of open clusters in our Galaxy are of order $10^{5}$.

Globular clusters are generally much more massive systems, containing up to of order $10^{6}$ stars, and in our Galaxy nearly all globular clusters are older than $\sim 10$ Gyr. (Globular clusters 
Table 1: Typical Open and Globular Cluster Characteristics (adapted from [9])

\begin{tabular}{lcc}
\hline \hline & Open & Globular \\
\hline Total Mass $\left(\mathrm{M}_{\odot}\right)$ & $10^{2}-10^{3}$ & $10^{4}-10^{6}$ \\
Central Density $\left(\mathrm{M}_{\odot} \mathrm{pc}^{-3}\right)$ & $10-10^{3}$ & $10^{4}-10^{5}$ \\
Central Velocity Dispersion $\left(\mathrm{km} \mathrm{s}^{-1}\right)$ & $0.1-1$ & $1-10$ \\
Half-mass Radius $(\mathrm{pc})$ & $1-4$ & $1-5$ \\
Binary Fraction & $0.1-0.5$ & $0.01-0.1$ \\
Age (Gyr) & $0.001-$ few & $10-12$ \\
Half-mass Relaxation Time $(\mathrm{Myr})$ & $15-250$ & $60-4500$ \\
Encounter Time at $\leq 1$ AU $(\mathrm{Myr})$ & $<1->$ cluster age & $<1-100$ \\
\hline
\end{tabular}

are not forming today in our Galaxy, though similar systems are forming elsewhere). Their central densities are generally much higher than open clusters, with a typical value of $10^{4} \mathrm{M}_{\odot} \mathrm{pc}^{-3}$. Therefore, all else being equal, the time between stellar encounters in a typical globular cluster is expected to be much shorter than in most open clusters (though the encounter time also depends inversely on both the binary frequency and the mean binary semi-major axis, both of which can be much larger in open clusters [58], see also Table 1). There are about 150 known globular clusters in our Galaxy.

In Table 1, I compare the typical properties of open and globular clusters. Many of these values should be self explanatory, given the name and units. For clarity, the half-mass radius is a typical measurement of the size of a star cluster, and is defined as the distance from the cluster center inside of which resides half of the mass of the cluster. We generally quantify the frequency (or fraction) of binaries as $f_{\mathrm{b}}=N_{\mathrm{b}} /\left(N_{\mathrm{s}}+N_{\mathrm{b}}+\ldots\right)$, where $N_{\mathrm{b}}$ is the number of binaries, $N_{\mathrm{s}}$ is the number of single stars, and “..." signifies higher-order multiples. In calculating the half-mass relaxation times, I follow [89] and assume a mean stellar mass of $0.5 \mathrm{M}_{\odot}$ and set the Coulomb logarithm, $\ln (\Lambda)=\ln (\lambda N)$, where $N$ is the number of stars and $\lambda=0.1$. Finally the encounter times show estimates of the mean time between any single-single, single-binary or binary-binary encounters in the cluster at an impact parameter of $\leq 1 \mathrm{AU}$ (using equations from [58]).

\section{A Short Primer on Star Cluster Dynamics and The Role of Binary Stars}

The internal dynamical evolution of star clusters is often conceptualized, at a basic level, as being governed by two primary processes. The first is known as "two-body relaxation", and is the combination of the long-range cumulative effects of weak stellar encounters. Through two-body relaxation, stars gradually exchange energy, which evolves the cluster toward thermal equilibrium, and drives the processes of evaporation, mass segregation and core collapse, on a typical time-scale of a half-mass relaxation time. Early models of star cluster evolution applying this principle discovered that without any additional energy source, star clusters should undergo a runaway collapse toward an infinite central density, known as the gravothermal "catastrophe" or instability $[6,61,17]$.

Speaking in thermodynamic terms, a self-gravitating system has a negative specific heat. "Heat" (kinetic energy) flows from the core, where the velocity dispersion is highest, to the halo 
through two-body relaxation. In such a system, if one takes heat away from the core, the core shrinks and gets hotter. Conversely, if one adds heat to the halo, it expands and grows colder (or at least does not heat up as quickly as the core). Therefore the temperature gradient steepens, and a runaway process is initiated.

In real star clusters, this "catastrophe" is averted by the second process that governs star cluster evolution, namely short-range strong stellar encounters between individual stars and binaries (and higher-order stellar systems). Early star cluster simulations showed that including the effect of binaries as an energy source (and even a single massive binary) can allow the core to rebound out of this collapsing phase [8]. These models also predict a new phase of gravothermal oscillations, where the core bounces between collapsing and expanding due to the interplay between the diffusion of energy out of the core, due to two-body relaxation processes, and the input of energy to the core, from close encounters with binary stars. More modern simulations show that with even a modest population of primordial binaries, most star clusters can actually avoid the initial dramatic core collapse phase for a Hubble time, due to "binary burning", where within a core binary population, any close binary can provide energy to the cluster until it is either ejected or the component stars merge [26].

Binaries are also self-gravitating systems that can be understood with a similar thermodynamic argument. If one takes energy away from a binary, for instance through a dynamical encounter with a passing star that has less kinetic energy than the binary binding energy, the binary will become tighter (i.e., reduce its semi-major axis) and therefore more tightly bound. These binaries are typically referred to as "hard" binaries [41]. A hard binary can donate energy through such encounters until the two stars merge (or until the semi-major axis is so small that encounters with other stars becomes sufficiently rare). The process of tightening or "hardening" of binaries may also be responsible for the production of many types of exotic stellar objects, and I return to this below. "Soft" binaries, on the other hand, have smaller binding energies than the typical kinetic energy of cluster stars, and therefore an encounter involving a soft binary can often completely unbind the binary.

Externally, star clusters are also subject to the Galactic potential, which will tidally strip stars that have appropriate velocities and reside toward the edge of a cluster (i.e., beyond the "tidal radius") [49]. Star clusters on Galactic disk crossing orbits or highly eccentric orbits can also experience shocks that can remove large amounts of stars in a short duration, relative to the cluster's orbital period within the Galaxy [97, 62]. Due to these external stripping mechanism, internal dynamical relaxation and stellar ejections, and the loss of stars shortly after the expulsion of natal gas (mentioned in Section 1.2), all star clusters are evaporating, and constantly seeding the Galactic field with their lost stellar and planetary systems.

\section{Empirical Characteristics of Multi-Star and Planetary Systems}

We now know that binary, and higher-order multiple stars are ubiquitous, and comprise a relatively large fraction of the stars in star forming regions [36, 51, 53, 7, 84], open clusters [66, $72,67,71,35,34,43$ ] and the Galactic field [83]. Interestingly, prior to a seminal paper by Hut et al. in 1992 [46], nearly all computer models of star clusters began with no binary stars (or higher-order multiples), due both to the previous lack of observational evidence and the computational expense 
of including primordial binaries (though in these models, binaries were generally allowed to form by tidal capture and/or three-body encounters in cluster cores). For an excellent and thorough review of our current observational understanding of stellar multiplicity, I recommend Duchêne \& Kraus (2013, [23]), and I summarize some of the more salient empirical results related to this paper below.

In general, we hope to derive from observations of single and binary stars, the binary frequency and distributions of binary orbital periods, eccentricities and mass-ratios (and perhaps other more difficult to measure orbital elements and individual masses). The most relevant of these orbital parameters for our discussion is the orbital period, which can be converted to a semi-major axis, and is a key component of the cross section for encountering another object, and the encounter rate.

Thanks to decades of observations and dedicated observers, we know a great deal about the field solar-type binary stars. The solar-type binary frequency in the Galactic field is approximately 50\% [83], and furthermore, distributions of orbital periods, eccentricities and mass ratios are known. For solar-type stars in the Galactic field, the orbital period $(P)$ distribution is observed to be $\log$-normal with a mean of $\log P=5.03$ and $\sigma_{\log P}=2.28$ (and here $P$ is in days) [83].

Furthermore, roughly $10 \%$ of solar-type field stars in the Solar Neighborhood are in triples or higher-order systems $[83,93]$. About $60 \%$ of known spectroscopic binaries (i.e., those with relatively short periods) in the Solar Neighborhood have additional companions [94, 4], and this fraction rises toward shorter inner binary orbital periods. For spectroscopic binaries with orbital periods $<3$ days, $96 \%$ have additional companions [94]. It is worth noting here explicitly that Andrei Tokovinin, in particular, has been a major contributor to our empirical understanding of triples and higher-order stellar systems in the Solar Neighborhood (e.g., [95, 94, 93, 92]).

A handful of Galactic open clusters also have sufficient observations to study statistically robust samples of binary stars. The study of binaries in open clusters is a major focus of the ongoing WIYN Open Cluster Study (WOCS, [63]), who use primarily radial velocities to study solar-type stars in open clusters. Observations of such open cluster stars suggest that binary frequencies and distributions of orbital elements and mass ratios are consistent with similar binaries in the Galactic field (generally, out to a limiting orbital period of a few thousand days) [34, 33, 29], and results for additional WOCS clusters are forthcoming.

Much less is known empirically about the fraction of triple (or higher-order) stars in star clusters. Taurus-Auriga [53], Hyades [72], Pleiades [66, 11] and Praesepe [67, 10] (the usual suspects) are the only clusters to my knowledge that have published observations with a statistically meaningful sample of solar-type triple stars. All of these surveys have different levels of completeness, but the raw numbers from these surveys indicate triple fractions between a few to $10 \%$ in these clusters, which, again, appears to be consistent with similar stars in the Galactic field.

It is also worth noting here that for the binary and triple fractions observed in these particular open clusters, encounters involving triples may be the most frequent type of dynamical encounter [57]. Therefore, though I've focused in Section 2 on the impact of binary stars on star cluster evolution, and indeed (primordial) triples are typically excluded from star cluster models (due to computational limitations), triples may be very important contributors to the dynamical evolution of star clusters, and perhaps also the production of exotic stars that are thought to result from dynamical encounters.

Toward higher-mass stars, the binary frequency increases markedly. For the most massive 
stars, the binary frequency is observed to be about $70 \%$, and perhaps even higher [83, 85, 14]. Also unlike the solar-type stars, $\mathrm{O}$ stars apparently have a much stronger preference for close binaries. The orbital period distribution of O-type stars in open clusters can be described by a power law with an exponent of $\sim-0.5$ [85]. This (and their relatively short lifetimes) suggests that a smaller fraction of $\mathrm{O}$ stars will undergo dynamical encounters in star clusters than do solar-type stars. On the other hand, more than $70 \%$ of $\mathrm{O}$ stars are expected to exchange mass with a binary companion, and one-third of these cases will end in a merger of the two stars [85].

Toward lower-mass stars, the binary frequency drops below 50\%, and most studies agree that low-mass stars have a lower frequency of companions than do solar-type stars. Current empirical values for the fraction of $0.1-0.5 \mathrm{M}_{\odot}$ stars with companions is about $25 \%$ [20, 22], while some estimates reach up to $\sim 40 \%$ ([25]; though it is now generally believed that this larger fraction was inflated due to an overestimate in the frequency of wide companions). The period distribution of low-mass stars is currently somewhat poorly constrained, due to the relatively small number of companions in volume-limited (and nearly complete) samples. It appears that a log-normal distribution similar to that of the solar-type binaries (though perhaps with somewhat smaller mean and $\sigma$ value) may hold for separations $<500 \mathrm{AU}$, and at larger separations an Öpik-like (i.e., flat in the $\log$ ) distribution may hold $[42,21]$.

Turning to planets, Winn \& Fabrykcy (2015, [98]) recently wrote an excellent review on "The Occurrence and Architecture of Exoplanetary Systems". Currently observations searching for and characterizing exoplanets are most complete for solar-type (FGK) stars (though of course no where near as complete in the orbital separation domain as for binary and multiple stars); within this sample, radial-velocity and transiting planet surveys predict that about $10 \%$ of stars have a giant planet at an orbital period of less than a few years, and about $50 \%$ have a compact system of smaller planets at orbital periods of less than one year [44, 27, 74]. From the Kepler survey, it appears that the orbital period distribution for planets around GK stars increases toward longer periods (out to a completeness limit of 50 days), for planets ranging from 2 to 32 Earth radii [45]. Microlensing surveys that probe wider separation systems (though often for lower-mass host stars) generally suggest that more than a quarter of stars have sub-Jovian planets orbiting at $\gtrsim 1$ AU $[40,16]$. Much less is known about exoplanets in star clusters, but current observations are consistent with the hypothesis that planets in clusters have a similar frequency as planets in the field (at least for the close separations probed by current observations, $[65,81,82,13])$.

In summary, observations indicate that the stellar companion fraction increases toward highermass stars, and for solar-type field stars, this fraction is about 50\%. The frequency of exoplanets around solar-type field stars is now known to also be quite high (and possibly $\gtrsim 50 \%$ ), thanks to the recent explosion of exoplanet observations. The distribution of binary-star orbital periods for all primary-star masses extends to very wide systems, to of order $10^{9}$ days or $10^{4-5} \mathrm{AU}$. The orbital period distribution for planets orbiting solar-type field stars increases with increasing periods, out to a completeness limit of 50 days, and wide-separation planets (i.e., $\gtrsim 1 \mathrm{AU}$ ) may also be quite common. Though the detection and characterization of stellar multiples and exoplanets in star clusters is generally less complete than for the field, current observations suggest that stars and planets may have similar characteristics both inside and outside of star clusters (at least toward the short-period end of the distribution). 


\section{Star Cluster Dynamics Affect Binaries and Planetary Systems}

The rate of encounters with other stars (or binaries) in star clusters increases with the size (i.e., orbital separation) of the target stellar or planetary system; therefore wider systems are expected to be more vulnerable to the dynamical effects of passing stars within a clustered birth environment. I list the general outcomes of dynamical encounters in Section 1, though disentangling the impact of encounters on stellar and planetary systems is generally far more complicated. Such encounters are usually thought of as one object encountering another (e.g., a single star encountering a binary); however, especially in the open cluster regime, these "simple" encounters can often be interrupted by another object and produce a "mini cluster", with a more complex behavior, that then dissolves [30]. Furthermore, in multi-stellar and multi-planet systems, perturbations to a wide outer companion that slightly modifies its orbit can, in turn, affect closer in companions due to the subsequent internal dynamical evolution of the system.

On a more global level, there are a few key observations that we can use to search for the imprint of dynamical encounters (no matter how complex the encounters are). Both (i) the companion frequency and (ii) the distributions of orbital elements can be modified by the cumulative effect of many encounters (and the general two-body relaxation processes in star clusters) as well as the less frequent strong encounters. Also, (iii) some of the products of these encounters may be observable as exotic objects. I discuss the effects of stellar dynamics in star cluster on these observables below.

\subsection{Changes to the Companion Frequency}

The term "hard-soft boundary" is often used to describe the division between the tight, hard binaries and the wide, soft binaries, and is a function of the velocity dispersion, and therefore also proportional to the overall cluster mass. Moreover, if one assumes a set primordial population of binaries across all clusters, for the sake of argument let's assume a fixed primordial binary frequency and a primordial period distribution that extends beyond the hard-soft boundary in most clusters, then one expects that the binary frequency will decrease with increasing cluster mass. Indeed, this is observed for globular clusters $[88,50,56]$, and open clusters appear to have still larger binary frequencies (and lower masses) [34, 33, 29]. This result may be due to the disruption of binaries (or the truncation of disks around proto-stars) by stellar encounters early on in the lives of star clusters.

Taking this one step further, the binaries born in the denser cluster core are expected to be most severely affected by dynamical disruptions at early times (before halo binaries have time to orbit through the core). Observations of NGC 1818, a young ( 30 Myr) rich cluster in the Large Magellanic Cloud, may show this exact phenomenon, where the binary frequency decreases toward the core $[19,60]$ (the opposite of what is observed in most older star clusters, $[68,32]$ ). One explanation for this is that the core binaries experienced an early phase of rapid dynamical disruptions resulting from strong stellar encounters, thereby preferentially decreasing the binary frequency toward the cluster core [31].

Over time two-body relaxation and mass segregation effects become dominant, which cycle binaries through the core (where wider binaries are destroyed) and also cause the (hard) binaries to preferentially sink toward the core relative to the lower-mass single stars. Thus, the radial distribution of the binary frequency in star clusters may evolve with time, from one that decreases toward 


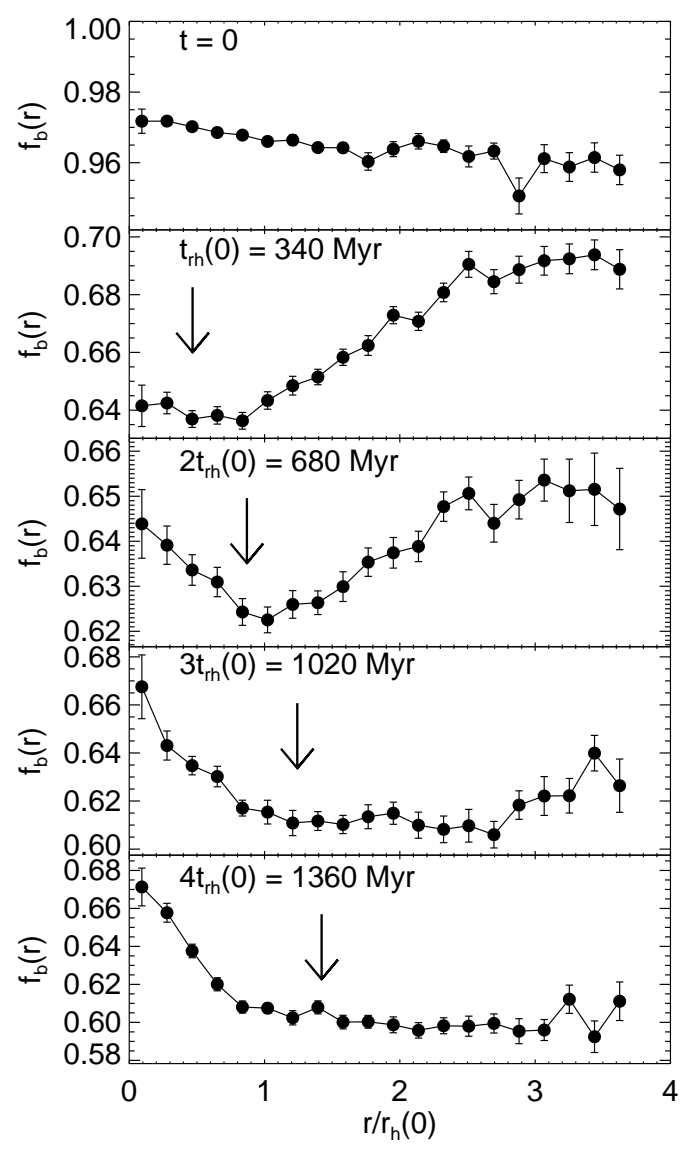

Figure 1: Radial distribution of the binary frequency for an $\mathrm{N}$-body model of the rich LMC star cluster NGC 1818, adapted from [31]. Each panel shows the distribution at a different age, from the initial conditions $(\mathrm{t}=0)$ at the top, and working downward in steps of the initial half-mass relaxation time $\left(t_{r h}(0)\right)$. The arrows in all but the top panel mark the respective cluster radii inside of which the local dynamical friction time-scale for a binary with a mass equivalent to that of the mean binary mass in the cluster is shorter than the simulated time, calculated analytically. Binaries at smaller radii than marked by the arrows are expected to sink toward the core due to dynamical friction and mass segregation effects.

the core (as in NGC 1818), to one that increases toward the core (as observed in most older star clusters). In Figure 1, I show an example of this phenomenon from a particular $N$-body model of the LMC star cluster NGC 1818 [28], with each panel showing the radial distribution of the binary frequency as a sequence in time (in steps of the initial half-mass relaxation time, $t_{\mathrm{rh}}(0)$ ). After one relaxation time, the global binary frequency drops from the initial value, due to the disruption of initially soft binaries, and this is most severe in the core. Then over time the radial distribution of the binary frequency evolves as described here. Importantly, these models (and others) show that the radial distribution of the binary frequency in a star cluster, may be an indicator of the cluster's "dynamical age" (i.e., how many relaxation times the cluster has lived through) .

Considering planetary systems in star clusters, most, if not all, planets would be "soft" by the definition given above (due to the small masses of planets as compared to stars). Therefore the concept of a hard-soft boundary as discussed above does not apply. However, $N$-body models do predict that the distribution of planet semi-major axes (or periods) should also be truncated by dynamical encounters [99], though here it is the encounter rate that sets the limit rather than the encounter energy.

\subsection{Changes to the Distributions of Orbital Elements}

The most dramatic change to the distributions of orbital elements predicted by numerical star 

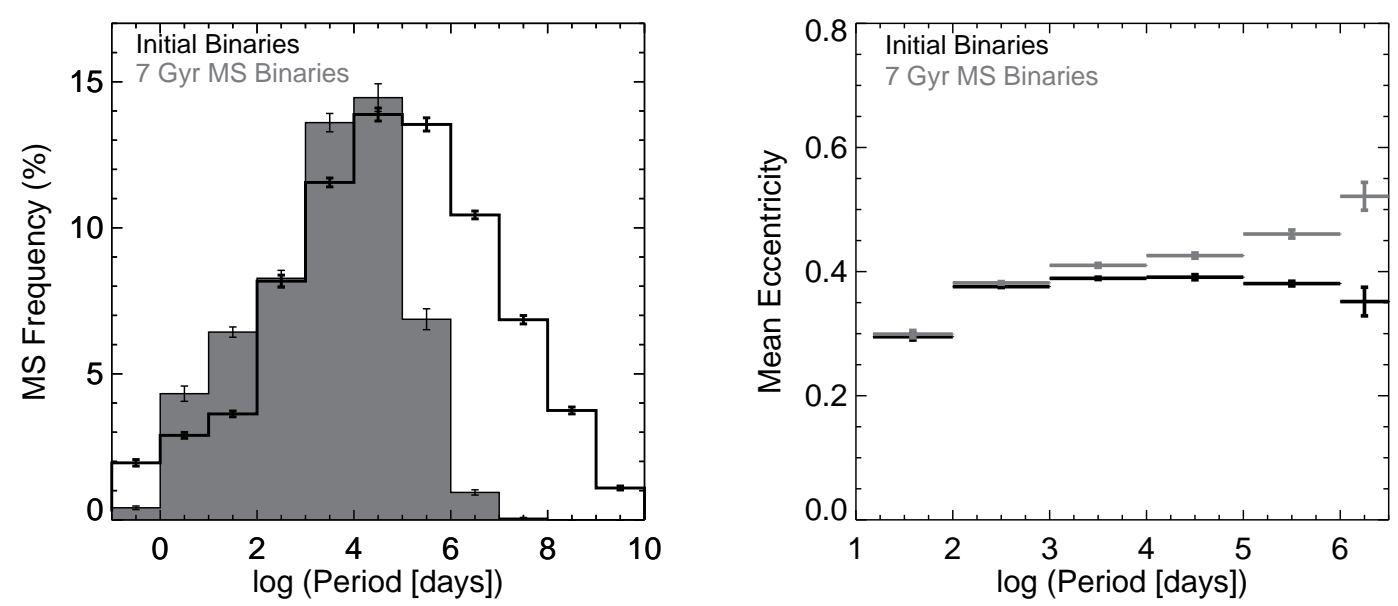

Figure 2: Changes to the main-sequence binary period (left) and eccentricity (right) distributions from an $N$-body model of the open cluster NGC 188, adapted from [32].

cluster models due to stellar dynamics is the steep drop off in the period distribution at the cluster hard-soft boundary, whereas, in the field, binaries of similar masses may exist out to much longer periods (see Figure 2). Unfortunately, observing this cutoff directly is very challenging for a number of reasons, and to my knowledge no statistically robust empirical evidence exists that can substantiate this model prediction (though there is little doubt that soft binaries are disrupted on the appropriate time-scales in clusters to create the cutoff). As a function of radius from the center of the cluster, models predict that for dynamically young clusters this hard-soft boundary will increase toward the cluster outskirts (where the density and velocity dispersion are lowest), until the cluster is sufficiently relaxed to cycle most binaries through the denser core [31].

Also for wider hard binaries, models predict an increase in eccentricity, resulting from perturbative fly-by encounters and exchanges [32]. These encounters may not disrupt the system, but tend to increase eccentricities most toward the widest systems, as these wider systems undergo encounters more frequently. Changes to the distribution of mass-ratios (the other common observable) are much more difficult to detect, as the mass-ratio is rarely a direct observable. Furthermore, changes to the mass-ratio distribution would result primarily from exchange encounters, which are far less frequent than perturbative fly-bys.

In Figure 2, I show an example of the changes to the eccentricity and period distributions due to stellar dynamics resulting from a particular $N$-body model of the open cluster NGC 188 [32]. Here the black lines and points show the initial conditions, and the grey region and points show the main-sequence binaries at the current cluster age (having lived through 7 Gyr of dynamical interactions). The drop in the period distribution at the hard-soft boundary, and the increase in eccentricities for the widest binaries are clearly visible. (However, note that these results combine 20 different $N$-body realizations of the cluster, and therefore the statistics are much better than could be expected for the true cluster, which contains of order 1000 stars.)

Much less work has been carried out to study these similar processes within a planetary population in star clusters. However, models do indicate that, like binary stars, the semi-major axis (or 
period) distribution for planets should be truncated by encounters (as also discussed above, [99]) and that eccentricities are most easily modified in planets with wider orbits [90].

\subsection{Formation of Exotic Stars}

Exotic stars and binary systems can also be formed through dynamical encounters within star clusters. A long-standing paradigm in the globular cluster community is that encounters involving hard binaries can shrink the binary orbits sufficiently to bring the stars into a stage of mass transfer (and potentially a merger). If one of the stars is a compact object, this can create an X-ray source (i.e. a CV, LMXB, etc.). Indeed the frequency of such X-ray sources is observed to be higher for star clusters with higher encounter rates [77].

Blue stragglers are another class of exotic stars, observed to be brighter and bluer than the main-sequence turnoff in an optical color magnitude diagram of a star cluster, that have long been associated with dynamical processes. Natalie Gosnell's contributions to these proceedings [37] discusses the origins of blue stragglers in detail. She finds that many, and perhaps most, of the blue stragglers in open clusters (and particularly those in the open cluster NGC 188, where we have the best observations) may come from a mass transfer mechanism involving giants [39, 38]. Moreover, though some blue stragglers likely have origins in stellar collisions (or perhaps mergers induced by binary hardening) the dominant blue straggler formation mechanism may not require dynamics. Furthermore, dynamical encounters may actually inhibit blue straggler formation by disrupting or modifying the orbits of (wide) binaries that would otherwise undergo mass transfer if evolved in isolation. More quantitatively, the specific frequency of blue stragglers in globular clusters decreases with increasing total absolute luminosity (mass) [75], and when also considering blue stragglers in dwarf galaxies and the field, the frequency of blue stragglers also decreases with increasing collision rate [79, 69]. Only in the most luminous (massive) globular clusters begin to produce blue stragglers predominantly through collisions [18]. These result therefore suggest that, though globular clusters are efficient at producing CVs and LMXBs through dynamics, similar encounters may prohibit the formation of blue stragglers.

"Sub-subgiants" (sometimes also termed "red stragglers") are another class of exotic star that may come from stellar collisions (among other mechanisms). These stars are observed to be redder than the main sequence but fainter than the subgiant branch in an optical color-magnitude diagram of a star cluster [3, 64], and are generally much less well studied than the others discussed here. In one collision scenario, the sub-subgiant may be a star observed right after a direct collision between two main-sequence stars, that is currently contracting and settling back into thermal equilibrium along an analogous track to pre-main-sequence stars [87]. In a second collision mechanism, a subsubgiant may be formed through a grazing collision between a subgiant and more compact star, that strips some of the envelope off of the subgiant (but does not result in a merger). We are actively working to model these channels in detail and to determine how likely these formation pathways are (and indeed other non-dynamical mechanisms may be more likely; see Emily Leiner's contribution to these proceedings, [59]). Nevertheless, our current models and calculation indicate that some sub-subgiants (and particularly those in globular clusters) may indeed be the products of stellar collisions brought on by dynamical encounters.

In summary observations suggest that for some exotic stars, dynamics may be very important (and perhaps critical) to their formation, while in other cases, dynamics may destroy or modify 
systems such that they do not form exotic stars when they would have otherwise. Regardless, the population of exotic stars in a star cluster is directly linked to stellar dynamics and the processes of binary-star evolution.

\section{Summary and Outlook}

In this review, I highlight the importance of stellar dynamical encounters in shaping the architectures of the multi-star and planet systems that we observe today. I argue that, because most stars are expected to form in star clusters or groups (some that are many orders of magnitude more dense than the Galactic field), that most binary (and higher-order-multiple) stars and planetary systems are subjected to encounters with other stars that can perturb their orbits and modify their primordial orbital elements and masses. Most of these stellar encounters are distant, though close energetic encounters do occur regularly in many clusters. Strong stellar encounters are most frequent and distant perturbations most severe for the widest systems, and therefore, as a population, these wide systems are affected most significantly. In multi-star and multi-planet systems, slight perturbations to an outer member can, over time, also propagate inwards through the system's internal dynamics.

Thus star clusters dynamically process their inhabitants through stellar encounters. Furthermore, star clusters are constantly losing stars to the Galactic field though various evaporation processes, and many star clusters dissolve completely within less than $1 \mathrm{Gyr}$ of birth. Therefore in order to properly interpret the observations of present-day stellar and planetary systems, both in star clusters and in the field, and especially if trying to map these observations back to study star and planet formation, we must consider the effects of dynamical encounters within a clustered birth environment.

Binary (and higher-order multiple) stars are also critical to the dynamical evolution of star clusters. It has long been known that binaries can reverse core collapse in globular clusters, and current models show that even a very modest binary frequency can delay the onset of core collapse in most globular clusters for over a Hubble time, through the processes of "binary burning". Furthermore, since encounters involving binaries (or higher-order systems) are far more common than those involving only single stars (because of the larger geometric cross-sections for multi-star systems), including binaries in star cluster models is critical. Moreover, star cluster models cannot produce realistic results without empirically motivated primordial populations of binaries, both in terms of the overall cluster evolution and the impacts of stellar encounters on the cluster population.

Looking ahead, I anticipate a few particularly important observational surveys for this field, namely WOCS, Kepler/K2, LSST and Gaia. The WOCS team are in the process of analyzing and publishing radial-velocity data for the solar-type binary populations in a set of key rich open clusters. Kepler has already discovered transiting planets in the open cluster NGC 6811 [65], and hopefully more similar discoveries will be forthcoming from the other open clusters in the original Kepler field and the many others in the K2 fields. LSST and Kepler/K2 will also surely discover and help to better characterize many eclipsing binary stars (and higher-order systems), and also to help identify exotic photometric variables that may be the result of dynamical encounters. Gaia parallaxes, proper motions, and to some extent also the radial velocities, will be an amazing resource for star cluster membership, dynamics and perhaps also binary star identifications. Taken 
together, over the coming decade or so, the depth and breadth (and uniformity) of empirical data for studying star cluster dynamics will dramatically increase.

High-performance computers, more efficient software parallelization, and more modular codes and code environments like AMUSE (amusecode.org, [78]), are pushing the computational limits on star cluster models into new regimes. Great strides have been made recently that may soon open the door to modeling realistic globular clusters with the star-by-star direct $N$-body method [96] (which is preferred as this requires the least simplifying assumptions of all methods). Realistic open cluster models including 1000s of stars and large populations of multi-planet and multi-stellar systems, evolved for many 100s of Myr, are also on the verge of feasibility and will be completed in the near future.

In summary, binaries are intimately linked to the dynamics of star clusters and the encounters that may modify binaries, higher-order-multiple stars and planetary systems within clustered birth environments. Advances in numerical modeling of star clusters will soon allow us to build very sophisticated star cluster models, that include realistic empirically guided stellar and planetary populations. Through these models and observations, we will study how and where previous stellar dynamical encounters from a clustered birth environment are imprinted on the stellar and planetary populations that we observe today.

\section{References}

[1] Adams, F. C., \& Myers, P. C., Modes of Multiple Star Formation, 2001, ApJ, 553, 744 [arXiv:astro-ph/0102039]

[2] Adams, F. C., The Birth Environment of the Solar System, 2010, ARA\&A, 48, 47 [arXiv:1001.5444]

[3] Albrow, M. D., Gilliland, R. L., Brown, T. M., et al., The Frequency of Binary Stars in the Core of 47 Tucanae, 2001, ApJ, 559, 1060 [arXiv:astro-ph/0105441]

[4] Allen, P. R., Burgasser, A. J., Faherty, J. K., \& Kirkpatrick, J. D., Low-mass Tertiary Companions to Spectroscopic Binaries. I. Common Proper Motion Survey for Wide Companions Using 2MASS, 2012, AJ, 144, 62 [arXiv:1206.4289]

[5] André, P., Belloche, A., Motte, F., \& Peretto, N., The initial conditions of star formation in the Ophiuchus main cloud: Kinematics of the protocluster condensations, 2007, A\&A, 472, 519 [arXiv:0706.1535]

[6] Antonov, V. A., Solution of the problem of stability of stellar system Emden's density law and the spherical distribution of velocities, Vestnik Leningradskogo Universiteta, Leningrad, 1962

[7] Bate, M. R., Stellar, brown dwarf and multiple star properties from a radiation hydrodynamical simulation of star cluster formation, 2012, MNRAS, 419, 3115 [arXiv:1110.1092]

[8] Bettwieser, E., A numerical method for the study of the gravothermal instability in star clusters, 1983, MNRAS, 203, 811

[9] Binney, J. \& Tremaine, S., Galactic Dynamics: Second Edition, Princeton University Press, Princeton, NJ, USA, 2008

[10] Bouvier, J., Duchêne, G., Mermilliod, J.-C., \& Simon, T., The formation and evolution of binary systems. III. Low-mass binaries in the Praesepe cluster, A\&A, 375, 989 [arXiv:astro-ph/0106493] 
[11] Bouvier, J., Rigaut, F., \& Nadeau, D., Pleiades low-mass binaries: do companions affect the evolution of protoplanetary disks?, 1997, A\&A, 323, 139

[12] Bressert, E., Bastian, N., Gutermuth, R., et al., The spatial distribution of star formation in the solar neighbourhood: do all stars form in dense clusters?, 2010, MNRAS, 409, L54 [arXiv:1009.1150]

[13] Brucalassi, A., Pasquini, L., Saglia, R., et al., Three planetary companions around M 67 stars, 2014, A\&A, 561, 9 [arXiv:1401.4905]

[14] Caballero-Nieves, S. M., Nelan, E. P., Gies, D. R., et al., A High Angular Resolution Survey of Massive Stars in Cygnus OB2: Results from the Hubble Space Telescope Fine Guidance Sensors, 2014, AJ, 147, 40 [arXiv:1311.5087]

[15] Cartwright, A., \& Whitworth, A. P., The statistical analysis of star clusters, 2004, MNRAS, 348, 589 [arXiv:astro-ph/0403474]

[16] Clanton, C. \& Gaudi, B. S., Synthesizing Exoplanet Demographics from Radial Velocity and Microlensing Surveys. II. The Frequency of Planets Orbiting M Dwarfs, 2014, ApJ, 791, 91 [arXiv:1404.7500]

[17] Cohn, H., Late core collapse in star clusters and the gravothermal instability, 1980, ApJ, 242, 765

[18] Davies, M. B., Piotto, G., \& de Angeli, F., Blue straggler production in globular clusters, 2004, MNRAS, 349, 129 [arXiv:astro-ph/0401502]

[19] de Grijs, R., Li, C., Zheng, Y., et al., Gravitational Conundrum? Dynamical Mass Segregation versus Disruption of Binary Stars in Dense Stellar Systems, 2013, ApJ, 765, 4 [arXiv:1301.1926]

[20] Delfosse, X., Beuzit, J.-L., Marchal, L., et al., M dwarfs binaries: Results from accurate radial velocities and high angular resolution observations, 2004, ASPC, 318, 166, Spectroscopically and Spatially Resolving the Components of the Close Binary Stars, ed. R. W. Hilditch, H. Hensberge \& K. Pavlovski

[21] Dhital, S., West, A. A., Stassun, K. G., \& Bochanski, J. J., Sloan Low-mass Wide Pairs of Kinematically Equivalent Stars (SLoWPoKES): A Catalog of Very Wide, Low-mass Pairs, 2010, AJ, 139, 2566 [arXiv:1004.2755]

[22] Dieterich, S. B., Henry, T. J., Golimowski, D. A., Krist, J. E., \& Tanner, A. M., The Solar Neighborhood. XXVIII. The Multiplicity Fraction of Nearby Stars from 5 to $70 \mathrm{AU}$ and the Brown Dwarf Desert around M Dwarfs, 2012, AJ, 144, 64 [arXiv:1206.1022]

[23] Duchêne, G. \& Kraus, A., Stellar Multiplicity, 2013, ARA\&A, 51, 269 [arXiv:1303.3028]

[24] Evans, N. J., II, Dunham, M. M., Jørgensen, J. K.,et al., The Spitzer c2d Legacy Results: Star-Formation Rates and Efficiencies; Evolution and Lifetimes, 2009, ApJS, 181, 321 [arXiv:0811.1059]

[25] Fischer, D. A. \& Marcy, G. W., Multiplicity among M dwarfs, 1992, ApJ, 396, 178

[26] Fregeau, J. M., Gürkan, M. A., Joshi, K. J., \& Rasio, F. A., Monte Carlo Simulations of Globular Cluster Evolution. III. Primordial Binary Interactions, 2003, ApH, 593, 772 [arXiv:astro-ph/0301521]

[27] Fressin, F., Torres, G., Charbonneau, D., et al., The False Positive Rate of Kepler and the Occurrence of Planets, 2013, ApH, 766, 81 [arXiv:1301.0842]

[28] Geller, A. M., de Grijs, R., Li, C., Hurley, J. R., Different Dynamical Ages for the Two Young and Coeval LMC Star Clusters, NGC 1805 and NGC 1818, Imprinted on Their Binary Populations, 2015, ApJ, 805, 11 [arXiv:1503.05198] 
[29] Geller, A. M., Latham, D. W., \& Mathieu, R. D., Stellar Radial Velocities in the Old Open Cluster M67 (NGC 2682). I. Memberships, Binaries, and Kinematics, 2015, AJ, 150, 97 [arXiv:1507.01949]

[30] Geller, A. M. \& Leigh, N. W. C., Interrupted Stellar Encounters in Star Clusters, 2015, ApJL, 808, 25 [arXiv:1506.08830]

[31] Geller, A. M., de Grijs, R., Li, C., \& Hurley, J. R., Consequences of Dynamical Disruption and Mass Segregation for the Binary Frequencies of Star Clusters, 2013, ApJ, 779, 30 [arXiv:1310.1085]

[32] Geller, A. M., Hurley, J. R., \& Mathieu, R. D., Direct N-body Modeling of the Old Open Cluster NGC 188: A Detailed Comparison of Theoretical and Observed Binary Star and Blue Straggler Populations, 2013, AJ, 145, 8 [arXiv:1210.1575]

[33] Geller, A. M. \& Mathieu, R. D., WIYN Open Cluster Study. XLVIII. The Hard-binary Population of NGC 188, 2012, AJ, 144, 54 [arXiv:1111.3950]

[34] Geller, A. M., Mathieu, R. D., Braden, E. K., et al., WIYN Open Cluster Study. XXXVIII. Stellar Radial Velocities in the Young Open Cluster M35 (NGC 2168), 2010, AJ, 139, 1383 [arXiv:1001.0033]

[35] Geller, A. M., Mathieu, R. D., Harris, H. C., \& McClure, R. D., WIYN Open Cluster Study. XXXII. Stellar Radial Velocities in the Old Open Cluster NGC 188, 2008, AJ, 135, 2264

[36] Ghez, A. M., Neugebauer, G., \& Matthews, K., The multiplicity of T Tauri stars in the star forming regions Taurus-Auriga and Ophiuchus-Scorpius: A 2.2 micron speckle imaging survey, 1993, AJ, 106, 2005

[37] Gosnell, N., Blue Straggler Stars: A Window Into Alternative Pathway Stellar Products, in Proceedings of Frank N. Bash Symposium 2015

[38] Gosnell, N., Mathieu, R. D., Geller, A. M., et al., Implications for the Formation of Blue Straggler Stars from HST Ultraviolet Observations of NGC 188, 2015, ApJ, 814, 163 [arXiv:1510.04290]

[39] Gosnell, N., Mathieu, R. D., Geller, A. M., et al., Detection of White Dwarf Companions to Blue Stragglers in the Open Cluster NGC 188: Direct Evidence for Recent Mass Transfer, 2014, ApJL, 783, 8 [arXiv:1401.7670]

[40] Gould, A., Dong, S., Gaudi, B. S., et al., Frequency of Solar-like Systems and of Ice and Gas Giants Beyond the Snow Line from High-magnification Microlensing Events in 2005-2008, 2010, ApJ, 720, 1073 [arXiv:1001.0572]

[41] Heggie, D. C., Binary evolution in stellar dynamics, 1975, MNRAS, 173, 729

[42] Henry, T. J., Jao, W.-C., Subasavage, J. P., et al., The Solar Neighborhood. XVII. Parallax Results from the CTIOPI $0.9 \mathrm{~m}$ Program: 20 New Members of the RECONS 10 Parsec Sample, 2006, AJ, 132, 2360 [arXiv:astro-ph/0608230]

[43] Hole, K. T., Geller, A. M., Mathieu, R. D., et al., WIYN Open Cluster Study. XXIV. Stellar Radial-Velocity Measurements in NGC 6819, 2009, AJ, 138, 159 [arXiv:0902.4040]

[44] Howard, A. W., Marcy, G. W., Johnson, J. A., et al., The Occurrence and Mass Distribution of Close-in Super-Earths, Neptunes, and Jupiters, 2010, Science, 330, 653 [arXiv:1011.0143]

[45] Howard, A. W., Marcy, G. W., Bryson, S. T., et al., Planet Occurrence within 0.25 AU of Solar-type Stars from Kepler, 2012, ApJS, 201, 15 [arXiv:1103.2541]

[46] Hut, P., McMillan, S., Goodman, J., et al., Binaries in globular clusters, 1992, PASP, 104, 981 
[47] Kaib, N. A., \& Raymond, S. N., Very Wide Binary Stars as the Primary Source of Stellar Collisions in the Galaxy, 2014, ApJ, 782, 60 [arXiv:1309.3272]

[48] Kharchenko, N. V., Piskunov, A. E., Schilbach, E., Röser, S., \& Scholz, R.-D., Global survey of star clusters in the Milky Way. II. The catalogue of basic parameters, 2013, A\&A, 558, 53 [arXiv:1308.5822]

[49] King, I., The structure of star clusters. I. an empirical density law, 1962, AJ, 67, 471

[50] Knigge, C., Leigh, N., \& Sills, A., A binary origin for 'blue stragglers' in globular clusters, 2009, Nature, 457, 288 [arXiv:0901.2345]

[51] Köhler, R., Kunkel, M., Leinert, C., \& Zinnecker, H., Multiplicity of X-ray selected T Tauri stars in the Scorpius-Centaurus OB association, 2000, A\&A, 356, 541

[52] Kraus, A. L., \& Hillenbrand, L. A., Spatial Distributions of Young Stars, 2008, ApJL, 686, L111, [arXiv:0809.0893]

[53] Kraus, A. L., Ireland, M. J., Martinache, F., \& Hillenbrand, L. A., Mapping the Shores of the Brown Dwarf Desert. II. Multiple Star Formation in Taurus-Auriga, 2011, ApJ, 731, 8 [arXiv:1101.4016]

[54] Lada, C. J., \& Lada, E. A., Embedded Clusters in Molecular Clouds, 2003, ARA\&A, 41, 57 [arXiv:astro-ph/0301540]

[55] Larson, R. B., Star formation in groups, 1995, MNRAS, 272, 213

[56] Leigh, N., Knigge, C., \& Sills, A., et al., The origins of blue stragglers and binarity in globular clusters, 2013, MNRAS, 428, 897 [arXiv:1210.0542]

[57] Leigh, N. \& Geller, A. M., Small-N collisional dynamics: pushing into the realm of not-so-small N, 2012, MNRAS, 425, 2369 [arXiv:1207.2469]

[58] Leigh, N., \& Sills, A., An analytic technique for constraining the dynamical origins of multiple star systems containing merger products, 2011, MNRAS, 410, 2370 [arXiv:1009.0461]

[59] Leiner, E., Geller, A. M., \& Mathieu, R. D., On the Origins of Sub-Subgiants: Mass Transfer, Dynamical Encounters, and Magnetic Fields, in Proceedings of Frank N. Bash Symposium 2015

[60] Li, C., de Grijs, R., \& Deng, L., The binary fractions in the massive young Large Magellanic Cloud star clusters NGC 1805 and NGC 1818, 2013, MNRAS, 436, 1497 [arXiv:1309.0929]

[61] Lynden-Bell, D. \& Wood, R., The gravo-thermal catastrophe in isothermal spheres and the onset of red-giant structure for stellar systems, 1968, MNRAS, 138, 495

[62] Madrid, J. P., Hurley, J. R., \& Martig, M., The Impact of Galaxy Geometry and Mass Evolution on the Survival of Star Clusters, 2014, ApJ, 784, 95 [arXiv:1402.2289]

[63] Mathieu, R. D., The WIYN Open Cluster Study, 2000, ASPC, 198, 517, Stellar Clusters and Associations: Convection, Rotation, and Dynamos, ed. R. Pallavicini, G. Micela, \& S. Sciortino

[64] Mathieu, R. D., van den Berg, M., Torres, G., et al., Sub-Subgiants in the Old Open Cluster M67?, 2003, AJ, 125, 246 [arXiv:astro-ph/0209568]

[65] Meibom, S., Torres, G., Fressin, F., et al., The same frequency of planets inside and outside open clusters of stars, 2013, Nature, 499, 55 [arXiv:1307.5842]

[66] Mermilliod, J.-C., Rosvick, J. M., Duquennoy, A., \& Mayor, M., Investigation of the Pleiades cluster. II - Binary stars in the F5-KO spectral region, 1992, A\&A, 265, 513 
[67] Mermilliod, J.-C. \& Mayor, M., Investigation of the Praesepe cluster III. Radial velocity and binarity of the F5-KO Klein-Wassink stars, 1999, A\&A, 352, 479 [arXiv:astro-ph/9911405]

[68] Milone, A. P., Piotto, G., Bedin, L. R., et al., The ACS survey of Galactic globular clusters. XII. Photometric binaries along the main sequence, 2012, A\&A, 540, 16 [arXiv:1111.0552]

[69] Momany, Y., The Blue Straggler Population in Dwarf Galaxies, 2015, 129, in Ecology of Blue Straggler Stars, Springer-Verlag Berlin Heidelberg [arXiv:1406.3472]

[70] Parker, R, J., Goodwin, S. P., \& Allison, R. J., The evolution of binary populations in cool, clumpy star clusters, 2011, MNRAS, 418, 2565 [arXiv:1108.3566]

[71] Patience, J., Ghez, A. M., Reid, I. N., \& Matthews, K., A High Angular Resolution Multiplicity Survey of the Open Clusters Î́s Persei and Praesepe, 2002, AJ, 123, 1570 [arXiv:astro-ph/0111156]

[72] Patience, J., Ghez, A. M., Reid, I. N., Weinberger, A. J., \& Matthews, K., The Multiplicity of the Hyades and Its Implications for Binary Star Formation and Evolution, 1998, AJ, 115, 1972 [arXiv:astro-ph/9801216]

[73] Peretto, N., André, P., \& Belloche, A., Probing the formation of intermediate- to high-mass stars in protoclusters. A detailed millimeter study of the NGC 2264 clumps, 2006, A\&A, 445, 979 [arXiv:astro-ph/0508619]

[74] Petigura, E. A., Howard, A. W. \& Marcy, G. W., Prevalence of Earth-size planets orbiting Sun-like stars, 2013, PNAS, 110, 19273 [arXiv:1311.6806]

[75] Piotto, G., De Angeli, F., King, I. R., et al., Relative Frequencies of Blue Stragglers in Galactic Globular Clusters: Constraints for the Formation Mechanisms, 2004, ApJL, 604, 109 [arXiv:astro-ph/0402592]

[76] Plummer, H. C., On the problem of distribution in globular star clusters, 1911, MNRAS, 71, 460

[77] Pooley, D., Lewin, W. H. G., Anderson, S. F., et al., Dynamical Formation of Close Binary Systems in Globular Clusters, 2003, ApJ, 591, 131 [arXiv:astro-ph/0305003]

[78] Portegies Zwart, S., McMillan, S. L. W., van Elteren, E., Pelupessy, I., \& de Vries, N., Multi-physics simulations using a hierarchical interchangeable software interface, 2013, CoPhC, 183, 456 [arXiv:1204.5522]

[79] Preston, G. W. \& Sneden, C., What Are These Blue Metal-Poor Stars?, 2000, AJ, 120, 1014

[80] Proszkow, E.-M., Adams, F. C., Hartmann, L. W., \& Tobin, J. J., Kinematic Signatures of Subvirial Initial Conditions in Young Clusters, 2009, ApJ, 697, 1020 [arXiv:0903.3242]

[81] Quinn, S. N., White, R. J., Latham, D. W., et al., Two "b"s in the Beehive: The Discovery of the First Hot Jupiters in an Open Cluster, 2012, ApJL, 756, 33 [arXiv:1207.0818]

[82] Quinn, S. N., White, R. J., Latham, D. W., et al., HD 285507b: An Eccentric Hot Jupiter in the Hyades Open Cluster, 2014, ApJ, 787, 27 [arXiv:1310.7328]

[83] Raghavan, D., McAlister, H. A., Henry, T. J., et al., A Survey of Stellar Families: Multiplicity of Solar-type Stars, 2010, ApJS, 190, 1 [arXiv:1007.0414]

[84] Sana, H., de Koter, A., de Mink, S. E., et al., The VLT-FLAMES Tarantula Survey. VIII. Multiplicity properties of the O-type star population, 2013, A\&A, 550, 107 [arXiv:1209.4638]

[85] Sana, H., de Mink, S. E., de Koter, A., et al.,Binary Interaction Dominates the Evolution of Massive Stars, 2012, Science, 337, 444 [arXiv:1207.6397] 
[86] Sánchez, N., \& Alfaro, E. J., The Spatial Distribution of Stars in Open Clusters, 2009, ApJ, 696, 2086 [arXiv:0902.1071]

[87] Sills, A., Lombardi, J. C., Jr., Bailyn, C. D., et al., Evolution of Stellar Collision Products in Globular Clusters. I. Head-on Collisions, 1997, ApJ, 487, 290 [arXiv:astro-ph/9705019]

[88] Sollima, A., Lanzoni, B., Beccari, G., Ferraro, F. R., \& Fusi Pecci, F., The correlation between blue straggler and binary fractions in the core of Galactic globular clusters, 2008, A\&A, 481, 701 [arXiv:0801.4511]

[89] Spitzer, L., Dynamical evolution of globular clusters, Princeton University Press, Princeton, NJ, USA, 1987

[90] Spurzem, R., Giersz, M., Heggie, D. C., Lin, D. N. C., Dynamics of Planetary Systems in Star Clusters, 2009, ApJ, 697, 458 [arXiv:astro-ph/0612757]

[91] Tobin, J. J., Hartmann, L., Furesz, G., Mateo, M., \& Megeath, S. T., Kinematics of the Orion Nebula Cluster: Velocity Substructure and Spectroscopic Binaries, 2009, ApJ, 697, 1103 [arXiv:0903.2775]

[92] Tokovinin, A., From Binaries to Multiples. I. Data on F and G Dwarfs within 67 pc of the Sun, 2014, AJ, 147, 86 [arXiv:1401.6825]

[93] Tokovinin, A., From Binaries to Multiples. II. Hierarchical Multiplicity of F and G Dwarfs, 2014, AJ, 147, 87 [arXiv:1401.6827]

[94] Tokovinin, A., Thomas, S., Sterzik, M., \& Udry, S., Tertiary companions to close spectroscopic binaries, 2006, A\&A, 450, 681 [arXiv:astro-ph/0601518]

[95] Tokovinin, A. A., MSC - a catalogue of physical multiple stars, 1997, A\&AS, 124, 75

[96] Wang, L., Spurzem, R., Aarseth, S., et al. NBODY6++GPU: ready for the gravitational million-body problem, 2015, MNRAS, 450, 4070 [arXiv:1504.03687]

[97] Webb, J. J., Harris, W. E., Sills, A., \& Hurley, J. R., The Influence of Orbital Eccentricity on Tidal Radii of Star Clusters, 2013, ApJ, 764, 124 [arXiv:1301.0626]

[98] Winn, J. N. \& Fabrycky, D. C., The Occurrence and Architecture of Exoplanetary Systems, 2015, ARA\&A, 53, 409 [arXiv:1410.4199]

[99] Zheng, X., Kouwenhoven, M. B. N., \& Wang, L., The dynamical fate of planetary systems in young star clusters, 2015, MNRAS, 453, 2759 [arXiv:1508.01593] 